\title{
Thermodynamics of the quantum spin-S XXZ chain
}

O. Rojas ${ }^{1, a}$, S.M. de Souza ${ }^{1}$, E.V. Corrêa Silva ${ }^{2}$, and M.T. Thomaz ${ }^{3}$

1 Departamento de Ciências Exatas, Universidade Federal de Lavras, P.O. Box 37, CEP 37200-000, Lavras-MG, Brazil

2 Departamento de Matemática e Computação, Faculdade de Tecnologia, Universidade do Estado do Rio de Janeiro, Estrada Resende-Riachuelo s/nº ${ }^{\circ}$ Morada da Colina, CEP 27523-000, Resende-RJ, Brazil

3 Instituto de Física, Universidade Federal Fluminense, Av. Gal. Milton Tavares de Souza s/nº CEP 24210-340, Niterói - RJ, Brazil

Eur. Phys. J. B 46, 385 (2005)

Received 29 August 2005

Published online 21 September 2005 - (c) EDP Sciences, Società Italiana di Fisica, Springer-Verlag 2005

Due to an error the paper by O. Rojas et al. entitled "Thermodynamics of the quantum spin-S XXZ chain" (Vol. 46, page 385), has been reproduced with wrong figures. Following this page the article will be printed again with its original page numbers.

\footnotetext{
a e-mail: or@stout.ufla.br
} 


\title{
Thermodynamics of the quantum spin-S XXZ chain
}

\author{
O. Rojas ${ }^{1, a}$, S.M. de Souza ${ }^{1}$, E.V. Corrêa Silva ${ }^{2}$, and M.T. Thomaz ${ }^{3}$ \\ 1 Departamento de Ciências Exatas, Universidade Federal de Lavras, P.O. Box 37, CEP 37200-000, Lavras-MG, Brazil \\ 2 Departamento de Matemática e Computação, Faculdade de Tecnologia, Universidade do Estado do Rio de Janeiro, Estrada \\ Resende-Riachuelo s/nº, Morada da Colina, CEP 27523-000, Resende-RJ, Brazil \\ 3 Instituto de Física, Universidade Federal Fluminense, Av. Gal. Milton Tavares de Souza s/nº, CEP 24210-340, Niterói - RJ, \\ Brazil
}

Received 8 September 2004 / Received in final form 18 April 2005

Published online 18 August 2005 - (C) EDP Sciences, Società Italiana di Fisica, Springer-Verlag 2005

\begin{abstract}
The thermodynamics of the spin- $S$ anisotropic quantum $X X Z$ chain with arbitrary value of $S$ and unitary norm, in the high-temperature regime, is reported. The single-ion anisotropy term and the interaction with an external magnetic field in the $z$-direction are taken into account. We obtain, for arbitrary value of $S$, the $\beta$-expansion of the Helmholtz free energy of the model up to order $\beta^{6}$ and show that it actually depends on $\frac{1}{S(S+1)}$. Its classical limit is obtained by simply taking $S \rightarrow \infty$. At $h=0$ and $D=0$, our high temperature expansion of the classical model coincides with Joyce's exact solution [11]. We study, in the high temperature region, some thermodynamic quantities such as the specific heat and the magnetic susceptibility as functions of spin and verify for which values of $S$ those thermodynamic functions behave classically. Their finite temperature behavior is inferred from interpolation of their highand low-temperature behavior, and shown to be in good agreement with numerical results. The finite temperature behavior is shown for higher values of spin.
\end{abstract}

PACS. 05.30.Ch Quantum ensemble theory - 75.10.Jm Quantized spin models - 75.10.Hk Classical spin models

\section{Introduction}

One-dimensional and quasi-one-dimensional quantum chain models have been intensively investigated for a twofold reason. On one hand, for the algebraic properties of spin-1/2 chain models ( $X X Z$ and generalized ladder models) which, being integrable models, can be solved exactly by the powerful Bethe ansatz technique (see Refs. [1] and [2], respectively). On the other hand, for the existence of quasi one-dimensional magnetic systems that exhibit nearly ideal one-dimensional behavior for some interval of temperature. The thermodynamic properties of those magnetic systems have been described by spin- $S X X Z$ models for various values of spin, mixed-spin models, ladder models, etc. In particular, there are materials well described by higher spin values of the $X X Z$ models such as $\mathrm{CsVCl}_{3}$ and $\mathrm{CsVBr}_{3}(S=3 / 2)[3-5],\left(\mathrm{C}_{10} \mathrm{H}_{8} \mathrm{~N}_{2}\right) \mathrm{MnCl}_{3}$ $(S=2)[6]$ and $\left(\mathrm{CD}_{3}\right)_{4} \mathrm{NMnCl}_{3}(S=5 / 2)[7,8]$. Motivated by the discovery of these materials, the magnetic and thermodynamic properties of the ferro- and antiferromagnetic isotropic spin- $S X X Z$ model for higher values of $S$ have been studied numerically and analytically (e.g., the high temperature expansions; see Ref. [9] and references therein).

The classical limit $(S \rightarrow \infty)$ of the $X X Z$ model is exactly soluble and was originally solved by Fisher [10],

\footnotetext{
a e-mail: or@stout.ufla.br
}

in the isotropic regime, for a null external magnetic field and no single-ion anisotropy term. Its anysotropic case has been solved by Joyce [11], also in the absence of an external magnetic field and without a single-ion anisotropy term, by writing the Helmholtz free energy of the classical $X X Z$ model as an integral equation whose solution is the spheroidal wave function. Unfortunately, in the presence of an external magnetic field and/or the single-ion anisotropy parameter, the solution of the integral equation cannot be reduced to any known function.

Recently, Fukushima et al. [12] obtained the specific heat and the magnetic susceptibility per site of a ferromagnetic mixed-spin model, with two kinds of spins, $s$ and $S$, arranged alternatively and coupled by a Heisenberg-type nearest-neighbor exchange for arbitrary values of $s$ and $S$ at the isotropic point $(\Delta=1$ in Eq. (1)) and in the absence of external magnetic fields. They obtained numerical results through the exact diagonalization method and the analytical high temperature expansions. For $s=S$ their model becomes a one-dimensional spin- $S X X Z$ model for arbitrary value of $S$. Their high temperature expansion of the specific heat per site, in the absence of an external magnetic field, goes up to order $(\beta J)^{11}$ and their expansion of the magnetic susceptibility, also for a vanishing magnetic field, goes up to $(\beta J)^{7}$ with a singleion anisotropy term in one of the spins. In reference [9] we extended the results of reference [12], for spin values 
up to $S=4$, including a free parameter of anisotropy in the $z$-direction, a single-ion anisotropy term and a nonzero external magnetic field in the $\beta$-expansions of the Helmholtz free energies up to order $(J \beta)^{6}$.

Following reference [9], we also consider the Hamiltonian of the anisotropic spin- $S X X Z$ with a single-ion anisotropy term and in the presence of an external magnetic field,

$$
\mathbf{H}=\sum_{i=1}^{N} J^{\prime}\left(\mathbf{S}_{i}, \mathbf{S}_{i+1}\right)_{\Delta}-h^{\prime} S_{i}^{z}+D^{\prime}\left(S_{i}^{z}\right)^{2} .
$$

We use the notation: $\left(\mathbf{S}_{i}, \mathbf{S}_{i+1}\right)_{\Delta} \equiv S_{i}^{x} S_{i+1}^{x}+S_{i}^{y} S_{i+1}^{y}+$ $\Delta S_{i}^{z} S_{i+1}^{z}$. Here, $S_{i}^{x}, S_{i}^{y}$ and $S_{i}^{z}$ stand for the spin- $S$ matrices in the $i$-th site of the chain and norm $\sqrt{S(S+1)}$; $N$ is the number of sites in the periodic chain; $J^{\prime}$ is the exchange integral; $\Delta$ is the anisotropy constant in the $z$-direction; $h^{\prime}$ is the external magnetic field in the $z$-axis and $D^{\prime}$ is the single-ion anisotropy parameter.

Even in one spatial dimension, the solution of the quantum chain model (1) is more complex if we consider a finite arbitrary spin- $S$ (with $S \neq 1 / 2$ ) once the $X X Z$ model becomes a non-integrable model and cannot be solved by the thermodynamic Bethe ansatz technique.

Numerical approaches for higher spin values becomes more involved since there are more degrees of freedom to be handled. Certainly, the high temperature expansions are easily manipulated by symbolic computer languages and can be used as a reliable check for numerical calculations in the high temperature regime.

The aim of the present communication is to extend the results of reference [9] to arbitrary values of the spin $S$ in hamiltonian (1). In doing so, we are including in the results of reference [12] the effects of anisotropies in the $z$-direction of the $X X Z$ model in the presence of an external magnetic field. Having a high temperature expansion for the model, for any $S$, we can derive its classical limit in this regime of temperature. We want to re-obtain the classical results of the Heisenberg model by considering the quantum nature of the spin variable, in opposition to the known results in the literature where this limit is derived from a chain of classical spins. From the high temperature expansion of the Helmholtz free energy, we can also verify for which values of $S$ the anisotropic quantum $X X Z$ model, in the presence of an external magnetic field, is well described by its classical version, extending the results of reference [9]. In the present article, comparison of thermodynamic functions for different values of $S$ is performed at the same (high) $T$, whereas in reference [9], they were plotted as functions of the "rescaled" temperature $\tilde{T}=\frac{T}{S(S+1)}$.

This paper is organized as follows: in Section 2 we use the method developed in reference [13] and an interpolation technique to obtain the high-temperature series expansion of the Helmholtz free energy for the quantum spin- $s$ XXZ chain ( $s$ with unitary norm) up to order $\beta^{6}$. In order to check our analytic $\beta$-expansion, in Section 2.1 our results for the classical limit $(S \rightarrow \infty)$ are compared to the well-known isotropic [10] and anisotropic [11] classical
Heisenberg chains. In Section 2.2 our results for finite values of $S$ are explored to include the effect of the anisotropy parameter $\Delta$, the presence of $D^{\prime}$ and $h^{\prime}$ in the Hamiltonian (1) and the dependence of thermodynamic functions on $S$. In Section 3 we use Padé representatives to enhance our high-temperature expansions for the spin-s $X X Z$ model, extending them to lower temperatures. We do so by taking into account the known behavior at $T \sim 0$. For the specific heat we use the method presented in reference [14], whereas for the magnetic susceptibility we apply the DlogPadé approximant [15] (antiferromagnetic case) and the two-point Padé approximant [16] (ferromagnetic case). In Section 4 we present our conclusions. In Appendix A we show that the trace of powers of $S^{z}$ can be written as an expansion in the square of the norm of the spin at each site. Finally, in Appendix B we present the hightemperature expansion, up to order $\beta^{6}$, of the Helmholtz free energy of the spin- $s X X Z$ model $(|s|=1)$, with a single-ion anisotropy term and in the presence of an external magnetic field.

\section{The high temperature behavior of the quantum spin-s $X X Z$ chain}

We intend to study the thermodynamics of the Hamiltonian (1) for arbitrary spin, including its classical limit $(S \rightarrow \infty)$. In this limit, the thermodynamic functions diverge and to render them finite we define a rescaled spin operator $\mathbf{s} \equiv \mathbf{S} / \sqrt{S(S+1)}$. This rescaled spin operator has unitary norm for all values of $S$. Rewriting the Hamiltonian (1) in terms of $\mathbf{s}$ and redefining the parameters $J \equiv S(S+1) J^{\prime}, h \equiv \sqrt{S(S+1)} h^{\prime}$ and $D \equiv S(S+1) D^{\prime}$, we obtain

$$
\mathbf{H}=\sum_{i=1}^{N} J\left(\mathbf{s}_{i}, \mathbf{s}_{i+1}\right)_{\Delta}-h s_{i}^{z}+D\left(s_{i}^{z}\right)^{2} .
$$

This also describes the dynamics of the $X X Z$ model of a spin of unitary norm and $(2 S+1) z$-components. We continue to use the notation: $\left(\mathbf{s}_{i}, \mathbf{s}_{i+1}\right)_{\Delta} \equiv s_{i}^{x} s_{i+1}^{x}+$ $s_{i}^{y} s_{i+1}^{y}+\Delta s_{i}^{z} s_{i+1}^{z}$. Here, $s_{i}^{x}, s_{i}^{y}$ and $s_{i}^{z}$ stand for the spin- $s$ $(s=1 / 2,1,3 / 2, \cdots)$ rescaled matrices in the $i$-th site of the chain; $N$ is the number of sites in the periodic chain; and $\Delta$ is the anisotropy constant in the $z$-direction.

In order to evaluate the Helmholtz free energy for arbitrary values of spin, we first calculate its expansion for a set of spin values using the Hamiltonian (2). We apply the method of reference [13] to calculate those $\beta$-expansions for arbitrary values of $J, \Delta, h$ and $D$. We presented elsewhere [9] the Helmholtz free energy of Hamiltonian (1) for a number of spin values (semi-integer and integer) up to $S=4$. We use the interpolation method to extend our results for fixed values of $S$ to arbitrary spin value. For the sake of obtaining the $\beta$-expansion of any spin- $S$ quantum chain up to order $\beta^{6}$, having the expansions presented in reference [9] is not enough.

In order to derive the expansion of the Helmholtz free energy of the hamiltonian (1) up to order $\beta^{n}$ (for a survey of the method [13], cf. Sect. 2 of Ref. [9]), we have to 
calculate normalized traces, at each site, of the products of $m_{i}$ matrices from the set $\left\{S^{x}, S^{y}, S^{z}\right\}$, so that $m_{i} \leq$ $2(k+1)$ and $k$ ranges over all possible powers of $\beta, k=$ $1,2, \cdots, n$. Due to the commutation relation $\left[S^{x}, S^{y}\right]=$ $i S^{z}$ and to the fact that the products $S^{x} S^{y}$ and $S^{y} S^{x}$ can be written in terms of $S^{z},\left(S^{z}\right)^{2}$ and the identity matrix $\mathbf{1}$, then the normalized trace at each site is reduced to the calculation of traces of $\left(S^{z}\right)^{l}$, with $l \in N$.

In equation (A.1) we show that the $\operatorname{tr}\left(\left(S^{z}\right)^{l}\right)$, for even values of $l$, is a polynomial of degree $(l+1)$ in $S$. Therefore the polynomial in $S$ of highest degree at order $\beta^{n}$ is of order $2(n+1)$. In order to carry out the interpolation of the expansion up to order $\beta^{6}$, we calculated the $\beta$-expansion of the Helmholtz free energy from the spin $S=1 / 2$ up to $S=7$. Later, by inspection of the expansion in $S$ and in $\beta$, we verified that the expansion in $S$ can be rewritten in terms of $S(S+1)$, that is, in terms of the eigenvalue of a constant of motion (the square of the norm of the spin-S at each site). In Appendix A we show, for arbitrary value of spin, that $\operatorname{tr}\left[\left(S_{i}^{z}\right)^{2 l}\right], l=1,2 \cdots$, is a polynomial of degree $(l-1)$ in the parameter $S(S+1)$.

The thermodynamic properties of the quantum spin$S X X Z$ chain, in the high temperature region, can be obtained from its Helmholtz free energy. We present in Appendix B the coefficients of the Helmholtz free energy $\mathcal{W}_{s}(\beta)$ derived from the Hamiltonian (2), up to order $\beta^{6}$, for any value of $S(S=1 / 2,1,3 / 2, \cdots)$. In particular, the result of Appendix B is also valid at the isotropic point of the Hamiltonian (2) with $D=0$ and $h=0$, when the model has rotational symmetry. The $\beta$-expansion of the Helmholtz free energy of Hamiltonian (1) is easily obtained from result (B.3) (see Appendix B). Fukushima et al. [12] obtained the $\beta$-expansion of the specific heat and magnetic susceptibility per site of the latter model for arbitrary spin at the isotropic point and in the absence of an external magnetic field. Our results agree with theirs, up to order $\beta^{6}$.

The range of validity in $\beta$ of our high-temperature expansions obviously depends on the particular values given to the parameters of (2). The thermodynamic properties of the chain depend on the sign of the product $J \Delta$. We let $J>0$ and let $\Delta$ refer to either the ferromagnetic $(\Delta<0)$ or the antiferromagnetic $(\Delta>0)$ phases.

\subsection{Comparison with known classical limits $(S \rightarrow \infty)$}

An interesting check to our expansion (B.3) is to verify if it recovers known classical limits $(S \rightarrow \infty$ or $y \rightarrow 0$, where $\left.y=\frac{1}{S(S+1)}\right)$ of the $X X Z$ chain. The classical limit of the $X X Z$ chain of the Hamiltonian (2) with $h=0$ and $D=0$ has been solved by Joyce [11]; this model (including the anisotropic constant $\Delta$ in the Hamiltonian (2)) is exactly solvable and its Helmholtz free energy is given by the radial component of the spheroidal wave function

$$
\mathcal{W}_{\infty}(\mu ; \beta)=-\frac{1}{\beta} \ln \left(R_{0,0}(-i \beta J / \sinh \mu, \cosh \mu)\right),
$$

where $\mu=\tanh ^{-1}\left(\frac{1}{\Delta}\right)$ and $R_{0,0}$ is the first radial spheroidal wave function [17]. For $|\Delta|=1$, equation (3) becomes the well-known result obtained by
Fisher [10], whose explicit expression is $\mathcal{W}_{\infty}\left( \pm \frac{\pi}{2}, \beta\right)=$ $-\frac{1}{\beta} \ln [\sinh (\beta J) /(\beta J)]$.

The classical limit of the $\beta$-expansion (B.3) is obtained by letting $y=0$. In order to have a shorter $\beta$-expansion of the Helmholtz free energy at $y=0$, we take $D=0$, and obtain

$$
\begin{aligned}
\mathcal{W}_{\infty}(\Delta, & \tilde{h}, 0 ; J \beta)=\left(-\frac{1}{6} \tilde{h}^{2}-\frac{1}{9}-\frac{1}{18} \Delta^{2}\right)(J \beta) \\
& +\frac{1}{9} \Delta \tilde{h}^{2}(J \beta)^{2} \\
& +\left(\frac{1}{180} \tilde{h}^{4}-\frac{1}{1350}-\frac{7}{135} \Delta^{2} \tilde{h}^{2}\right. \\
& \left.-\frac{7}{2700} \Delta^{4}+\frac{2}{135} \tilde{h}^{2}+\frac{2}{225} \Delta^{2}\right)(J \beta)^{3} \\
& +\left(-\frac{2}{135} \Delta \tilde{h}^{2}-\frac{4}{225} \Delta+\frac{46}{2025} \Delta^{3}\right) \tilde{h}^{2}(J \beta)^{4} \\
& +\left(\frac{424}{42525} \Delta^{2} \tilde{h}^{2}+\frac{179}{9450} \Delta^{2} \tilde{h}^{4}-\frac{359}{42525} \Delta^{4} \tilde{h}^{2}\right. \\
& -\frac{2}{2835} \tilde{h}^{2}-\frac{1}{525} \tilde{h}^{4}-\frac{107}{2679075} \Delta^{6}+\frac{212}{893025} \Delta^{4} \\
& \left.-\frac{632}{893025} \Delta^{2}+\frac{422}{2679075}-\frac{1}{2835} \tilde{h}^{6}\right)(J \beta)^{5} \\
& +\left(\frac{16}{2835} \Delta \tilde{h}^{2}-\frac{248}{14175} \Delta^{3} \tilde{h}^{2}\right. \\
& +\frac{2566}{893025} \Delta^{5}+\frac{1264}{893025} \Delta-\frac{836}{178605} \Delta^{3} \\
& \left.+\frac{1}{525} \Delta \tilde{h}^{4}\right) \tilde{h}^{2}(J \beta)^{6}+\mathcal{O}\left((J \beta)^{7}\right)
\end{aligned}
$$

where $\tilde{h} \equiv h / J$.

From (4) we see that in the absence of the external magnetic field $(h=0)$ the Helmholtz free energy is an even function of the anisotropic parameter $\Delta$. Under this condition, the classical limit of the $X X Z$ model does not distinguish between the ferromagnetic and the antiferromagnetic phases in the region of high temperatures.

Figure 1a shows a comparison of our $\beta$-expansion of the Helmholtz free energy to the numerical solution of equation (3), for $h=0, D=0$ and $\Delta= \pm 0.5$. They are in good agreement in the high temperature region; the percental error between them at $k T / J=0.2$ is less than $2.8 \%$. Figure $1 \mathrm{~b}$ performs the same comparison, but at the isotropic point $(\Delta= \pm 1)$. We also have good agreement, but for higher temperatures; percental error is about $3 \%$ for $k T / J=0.4$.

In conclusion, the classical limit of the expansion (B.3) (for $y=0$ ) coincides with Joyce's solution (3) of the Helmholtz free energy of the Hamiltonian (2) in a larger interval of temperature than that which we call "high temperature region".

\subsection{The thermodynamics of the spin-s $X X Z$ model}

The greater the (finite) spin, the greater the number of degrees of freedom to be handled, thus the more involved 
it gets to compute thermodynamic properties of the quantum spin-S model. In contrast, for infinite values of spin, the calculation of those properties for the classical version of the model is simpler, since we can treat the spin as a vector that rotates continuously. It is interesting to know for which values of $S$ the quantum spin model can be well approximated by its classical version, at least in the high temperature region.

In reference [9] we compared various thermodynamic functions of the chain model at the Heisenberg point $(\Delta= \pm 1)$, in the absence of the single-ion anisotropy term and external magnetic fields. We verified, in the high temperature region, that thermodynamic functions like the magnetic susceptibility per site have a classical behavior, for $S \geq 2$. Our intention in this section is to extend the results of reference [9] in order to include in this comparison (quantum $\times$ classical behavior) of spin models with anisotropies in the $z$-direction $(\Delta \neq \pm 1$ and $D \neq 0)$ and in the presence of an external magnetic field. This analysis can be realized from the expansion (B.3) of $\mathcal{W}_{s}(\beta)$. The referred thermodynamic functions are all derived from the Hamiltonian (2).

Let $C_{s}(\beta)$ and $C_{S}(\beta)$ be the specific heat per site for the spin- $s$ model (with unitary norm) and for the spin- $S$ chain (with norm equal to $\sqrt{S(S+1)}$ ), respectively. The specific heat per site is calculated from a derivative of the Helmholtz free energy $\left(C(\beta)=-\beta^{2} \frac{\partial^{2}(\beta \mathcal{W}(\beta))}{\partial \beta^{2}}\right)$. From the results (B.2) we obtain,

$$
\begin{array}{r}
C_{s}(J, \Delta, h, D ; \beta)=C_{S}\left(J, \Delta, \sqrt{S(S+1)} h, D ; \frac{\beta}{S(S+1)}\right) \\
=C_{S}\left(\frac{J}{S(S+1)}, \Delta, \frac{h}{\sqrt{S(S+1)}}, \frac{D}{S(S+1)} ; \beta\right) .
\end{array}
$$

These results show that $C_{S}$ is a homogeneous function of zero degree for all temperatures. The $\beta$ - expansion of the specific heat derived from equations (B.3) satisfies equations (5) identically.

From the high temperature expansion of $C_{s}$ we verify that $C_{s}=-\left(\frac{D^{2}}{15 S(S+1)}-\frac{h^{2}}{3}-\frac{2}{9}-\frac{4 D^{2}}{45}-\frac{\Delta^{2}}{9}\right) \beta^{2}+\mathcal{O}\left(\beta^{3}\right)$. In the high temperature region, the $X X Z$ model presents a tail of the Schottky peak [18] $\left(C_{S c h} \propto \beta^{2}\right)$, for all values of $S$.

Figure 2 shows the specific heat per site as a function of $J \beta$ for distinct values of $S$, including the classical limit $(S \rightarrow \infty)$ of the Hamiltonian (2) and the relative percental error of this function for various values of $S$ in relation to the classical specific heat per site. We take $h / J=0.5$ and $D / J=0.7$. Figure 2a pictures the antiferromagnetic case $(\Delta=0.3)$ and $(2 \mathrm{c})$ the ferromagnetic case $(\Delta=$ $-0.3)$. Figures $2 \mathrm{~b}$ and $\mathrm{d}$ show the relative percental error, i.e., $\left|\frac{C_{\infty}-C_{s}}{C_{\infty}}\right| \times 100 \%$, of Figures $2 \mathrm{a}$ and c, respectively, where $C_{\infty}$ is the classical limit of the specific heat per site. In both cases, even the $S=2$ model behaves classically (within an error smaller than $2 \%$ ) up to $J \beta=0.3$. Within

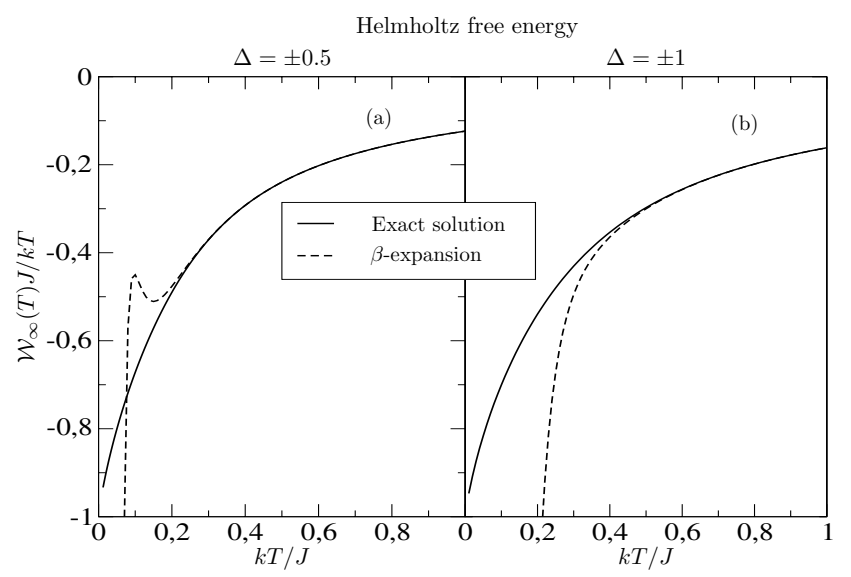

Fig. 1. The Helmholtz free energy $\mathcal{W}_{\infty}($ for $S \rightarrow \infty)$ per unit of $k T / J$ as a function of $k T / J$ for the classical $X X Z$ model in the absence of the single-ion anisotropy term $(D=0)$ and an external magnetic field $(h=0)$. In $(a)$ we plot the anisotropic cases with $\Delta= \pm 0.5$ and in (b) the isotropic cases $(\Delta= \pm 1)$. The solid line refers to the numerical solution of (3) and the dashed line to the expansion (4).

this range of error, we can also neglect the quantum nature of the spin model with $S \geq 5$ up to $J \beta \sim 1$.

Let $\chi_{s}(\beta)$ and $\chi_{S}(\beta)$ be the magnetic susceptibilities per site of the spin- $s$ model (with unitary norm) and of the spin- $S$ model (with norm equal to $\sqrt{S(S+1)}$ ), respectively. The relation between the magnetic susceptibility per site and the Helmholtz free energy is $\chi(\beta)=-\frac{\partial^{2} \mathcal{W}(\beta)}{\partial h^{2}}$. From the results (B.2) we obtain the relation between $\chi_{s}$ and $\chi_{S}$,

$\chi_{s}(J, \Delta, h, D ; \beta)=\chi_{S}\left(J, \Delta, \sqrt{S(S+1)} h, D ; \frac{\beta}{S(S+1)}\right)$

$$
=\frac{1}{S(S+1)} \chi_{S}\left(\frac{J}{S(S+1)}, \Delta, \frac{h}{\sqrt{S(S+1)}}, \frac{D}{S(S+1)} ; \beta\right) \text {. }
$$

Equating the r.h.s. of equations (6a) and (6b) we obtain that $\chi_{S}$ is a homogenous function of degree 1 for all values of temperature. Its $\beta$-expansion satisfies this property.

Figure 3 shows the magnetic susceptibility versus $J \beta$ for various finite values of $S$ and the classical chain model $(S \rightarrow \infty)$. Figure 3 b shows the relative percentage error of the magnetic susceptibility with respect to the classical curve for $S=1,3 / 2,4$ and 6 . In Figure 3 we have $\Delta=1$, $h / J=0.3$ and $D / J=0.5$. From Figure 3 b we verify that the magnetic susceptibility of the spin- $3 / 2$ can be approximated by the classical result up to $J \beta \sim 0.5$ with an error smaller than $2 \%$. The classical magnetic susceptibility curve is a good approximation of the spin- 2 model up to $J \beta \sim 0.7$.

Figure 4 shows the magnetization of the $X X Z$ model of the spin with unitary norm versus $h / J(M(\beta)=$ $\left.-\frac{\mathcal{W}(\beta)}{\partial h}\right)$. We call $M_{s}(\beta)$ the magnetization derived from 

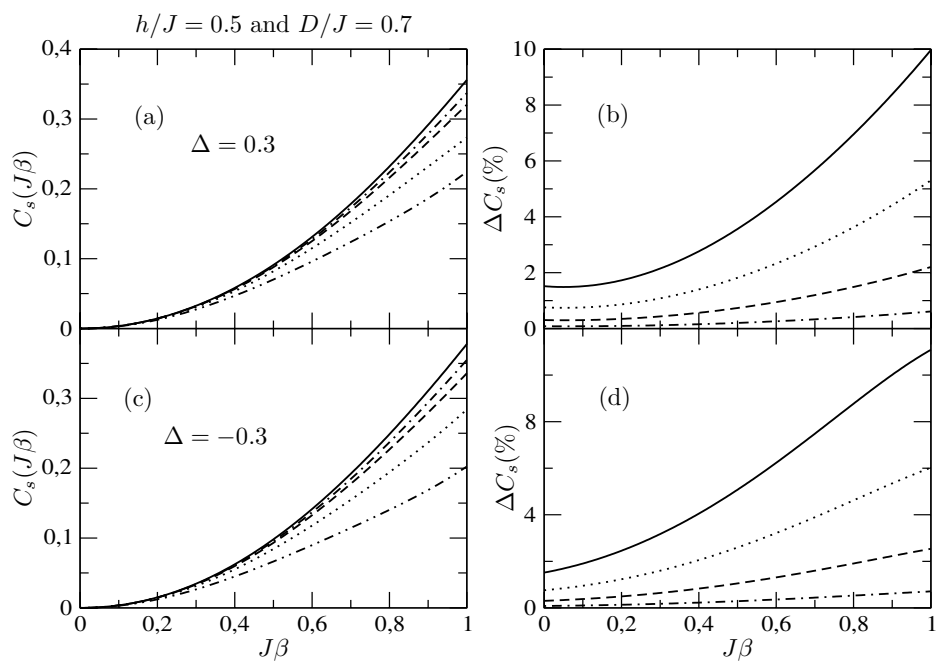

Fig. 2. Figures (a) and (c) show the specific heat $C_{s}(J \beta) ;(\mathrm{b})$ and $(\mathrm{d})$ show the relative percental errors between $C_{s}(\beta)$ and $C_{\infty}(\beta)$ (the classical limit) in (a) and $(c)$, respectively. For all plots we have $h / J=0.5$ and $D / J=0.7$. In (a) we let $\Delta=0.3$ and in (c) we have $\Delta=-0.3$. In (a) and (c), line styles correspond to different values of spin, as follows: $S=1 / 2$ (dashed and double-dotted lines), $S=1$ (dotted line), $S=2$ (dashed line), $S=3$ (dashed and dotted lines) and $S \rightarrow \infty$ (solid line). In (b) and (d) we have $S=2$ (solid line), $S=3$ (dotted line), $S=5$ (dashed line) and $S=10$ (dashed and dotted line).
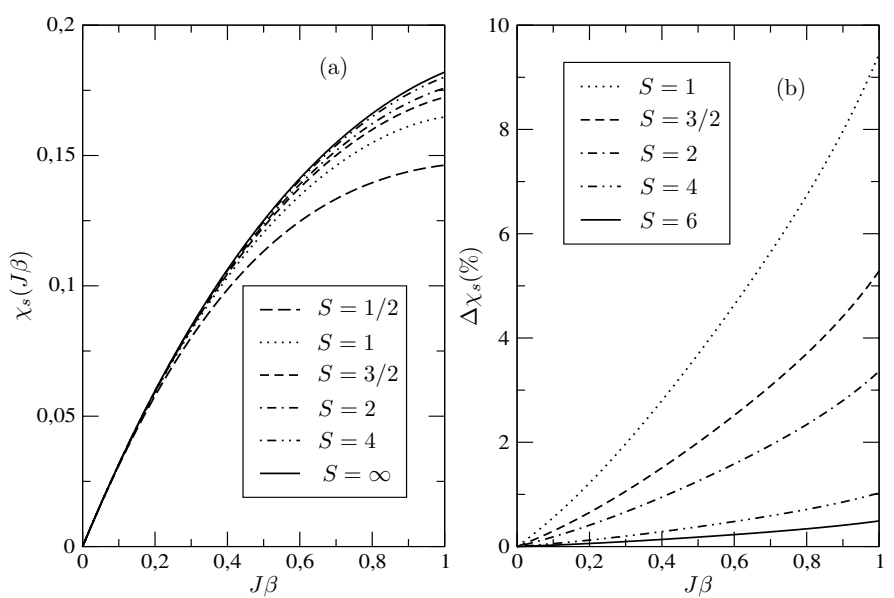

Fig. 3. (a) The magnetic susceptibility per site $\chi_{s}(J \beta)$ obtained from expansion (B.3) for various values of spin, including the classical limit (solid line). We take $\Delta=1, h / J=0.3$ and $D / J=-0.5$. (b) Relative percental error between $\chi_{s}$ and $\chi_{\infty}$, for several values of $S$.

Hamiltonian (2) and $M_{S}(\beta)$ the magnetization derived from (1). From equations (B.2), we also obtain

$$
\begin{aligned}
& M_{s}(J, \Delta, h, D ; \beta)= \\
& \quad \sqrt{S(S+1)} M_{S}\left(J, \Delta, \sqrt{S(S+1)} h, D ; \frac{\beta}{S(S+1)}\right) \\
& = \\
& \quad \frac{1}{\sqrt{S(S+1)}} M_{S}\left(\frac{J}{S(S+1)}, \Delta, \frac{h}{\sqrt{S(S+1)}}, \frac{D}{S(S+1)} ; \beta\right) .
\end{aligned}
$$

From equations (7) we obtain that $M_{S}$ is a homogeneous function of degree 1 at all temperatures. This condition is satisfied by the $\beta$-expansion of the magnetization, derived from equation (B.3).

Figure 4a shows the magnetization $M_{s}$ as a function of $h / J$ at $J \beta=0.4$. We choose the same values of constants in the Hamiltonian (2) as in Figure $3, \Delta=1$ and $D / J=$ -0.5 . Comparison of Figures $3 \mathrm{~b}$ and $4 \mathrm{~b}$ shows a closer similarity of quantum and classical magnetization curves (even for low values of spin such as $S=3 / 2$ ) than that of magnetic susceptibility curves, as far as high temperatures are concerned. 

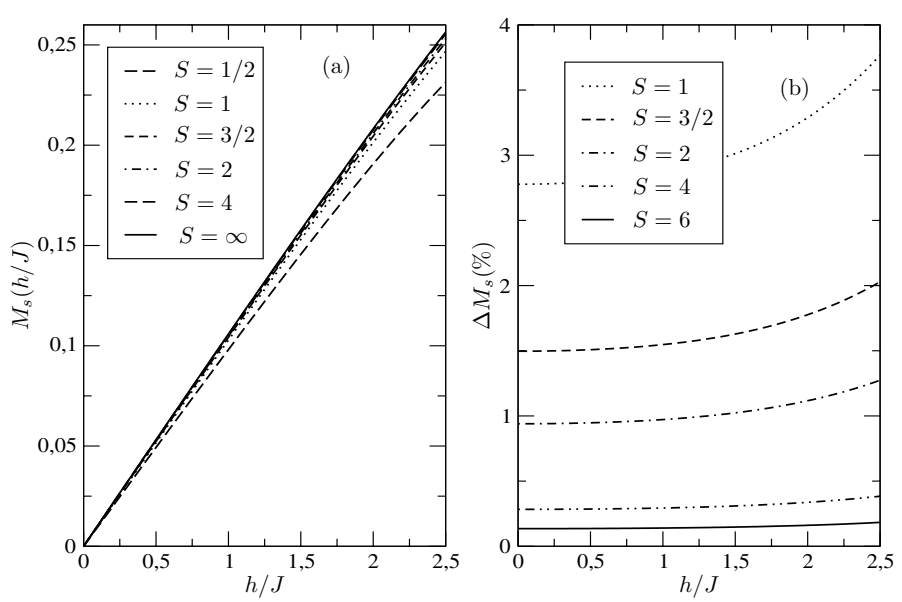

Fig. 4. (a) Magnetization $M_{s}(h / J)$ at $J \beta=0.4$, calculated from the $\beta$-expansion of $\mathcal{W}_{s}(\beta)$, for various values of spin, including the classical model $(S \rightarrow \infty)$. We take $\Delta=1$ and $D / J=-0.5$. (b) Relative percental error of $M_{s}$, for several values of $S$, with respect to $M_{\infty}$.
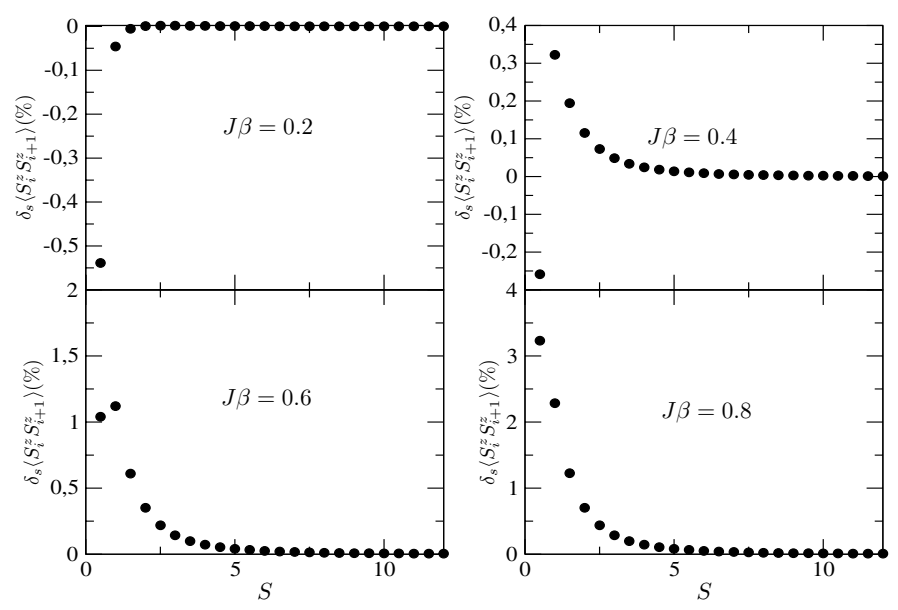

Fig. 5. Percental variation of the correlation function $\delta_{s}\left\langle S_{i}^{z} S_{i+1}^{z}\right\rangle$ as a function of $S$, for several values of $J \beta$ in the high temperature region. In all plots we take $\Delta=1, h / J=0.3$ and $D=-0.5$.

The correlation function of spin $z$-components between first neighbors is written as $\left\langle S_{i}^{z} S_{i+1}^{z}\right\rangle_{s}=\frac{\partial \mathcal{W}_{s}(\beta)}{\partial \Delta}$, for a given spin $s$. Its percental variation (when $s$ varies by halfinteger steps), shown in Figure 5, is defined as

$$
\delta_{s}\left\langle S_{i}^{z} S_{i+1}^{z}\right\rangle \equiv \frac{\left\langle S_{i}^{z} S_{i+1}^{z}\right\rangle_{(s+1 / 2)}-\left\langle S_{i}^{z} S_{i+1}^{z}\right\rangle_{s}}{\left\langle S_{i}^{z} S_{i+1}^{z}\right\rangle_{s}} .
$$

For $S \gg 1$, the function $\delta_{s}\left\langle S_{i}^{z} S_{i+1}^{z}\right\rangle$ has an expansion in $S^{-1}$ whose leading term is $S^{-3}$, and its coefficient depends on the temperature. In Figure 5 we take $\Delta=1, h / J=$ 0.3 and $D / J=-0.5$. In the very high temperature of $J \beta=0.2$, only the correlation functions for $S=1 / 2$ and $S=1$ differ from the classical curve by a difference smaller than $0.6 \%$. As we lower the temperature, spins of higher values have correlation functions between first neighbors that differ slightly from the classical ones, but even at $J \beta \sim 0.8$, the correlation function for $S=2$ approximates the classical result with an error smaller than $1 \%$.

\section{Extension of the thermodynamics of the quantum spin-s $X X Z$ chain to lower temperatures}

Although there are only seven terms in the hightemperature expansion of the Helmholtz free energy of the spin-s $X X Z$ model (see Appendix B), different Padé approximants permit us to enhance our analytic results and extend them to lower temperatures. In this section, we shall assume a quantum spin chain with unitary norm.

Bernu and Misguich [14] presented an approach to "interpolate" the high- and low-temperature behaviors of the 
specific heat per site, provided that the ground state energy per site of the chain is known. We refer the reader to their work for details on the method.

The $X X Z$ models (with $S \neq 1 / 2$ ), at the isotropic points $(\Delta \pm 1)$, are among the most studied ones. For this reason, in this section we calculate the Padé approximants at those points, for the sake of comparison of our results to the literature.

At $T \sim 0$ the behavior of the specific heat per site is distinct for gap and gapless models. Ferromagnetic and half-odd integer spin chains do not have excitation energy gaps and the specific heat per site around $T=0$ is $C_{s} \sim T^{p / q}$, where $p$ and $q$ are integers [14]. By contrast, the integer antiferromagnetic spin chains have an energy gap between the ground state energy and the lowest exited state and this thermodynamic function, in the region of $T \sim 0$, is $C_{s} \sim e^{-\frac{E_{g a p}}{k T}} T^{\alpha}[14,19,20]$, where $\alpha$ depends on the spin. Particularly for $S=1 \alpha$ is $-3 / 2$ [20], whereas for $S \geq 2$ its unknown. As the spin increases, this energy gap decreases rapidly; it only vanishes at the classical limit $(S \rightarrow \infty)$, though.

In reference [21], Yamamoto studied numerically the thermodynamics of the $S=2 X X Z$ model with $\Delta=1$, $D=0$ and $h=0$. The ground state energy per site of this model for the non-unitary spin chain was obtained in reference [22]. The ground state energy per site of the unitary spin chain can then be easily obtained, namely $e_{0} / J=-0.79354$. This model exhibits an energy gap $E_{\text {gap }} / J \approx 0.0149$ [22]. At very low temperatures it is expected that the specific heat vanish exponentially $\left(C_{s} \sim \exp \left(-E_{\text {gap }} / k T\right)\right)$ as $T \rightarrow 0$. On the other hand, the spin wave theory yields a linear behavior for the specific heat over a finite interval of intermediate low temperatures [23]. We compare the numerical results of Yamamoto [21] for the specific heat per site with our extended results for the antiferromagnetic spin- 2 chain at lower temperatures. The lowest value of $k T / J$ in reference [21] is 0.083 , which is larger than $E_{\text {gap }} / J$. Therefore, the available data for comparison refers to the intermediate low temperature region. In order to enhance our $\beta$-expansion of $C_{2}(T)$ to lower temperatures, we simply use $C_{2} \sim T$. The entropy at low temperatures behaves as $\mathcal{S}(e)=\left(e-e_{0}\right)^{1 / 2}$, where $e$ is the ground state energy per site, and it has an essential singularity when $e \rightarrow e_{0}$. Following reference [14], we construct an auxiliary function $\mathcal{G}(e) \equiv(\mathcal{S}(e))^{2}$, which is analytic in the interval $\left[e_{0}, 0\right]$, and use the Padé approximant to fit $\mathcal{G}(e)$, so the entropy becomes $\mathcal{S}(e)=\tilde{\mathcal{G}}(e)^{1 / 2}$. By $\tilde{\mathcal{G}}(e)$ we denote the Padé approximant to $\mathcal{G}(e)$. The specific heat per site of the model is obtained using the relation $C_{2}(e)=-\mathcal{S}^{\prime}(e)^{2} / \mathcal{S}^{\prime \prime}(e)$, which can be plotted in parametric form $\left\{T(e), C_{2}(e)\right\}$ (for details see Ref. [14]).

In Figure 6 (top) we compare all "acceptable" Padé approximants $P_{n, m}$ (i.e., all approximants so that $n+m=7$ and that do not possess spurious singularities) and their average $P_{a v r}$, to the numerical results obtained by the quantum Monte Carlo method (QMC) [21] and the $\beta$-expansion of $C_{2}(T)$. Figure 6 (bottom) displays the percental deviation of each Padé

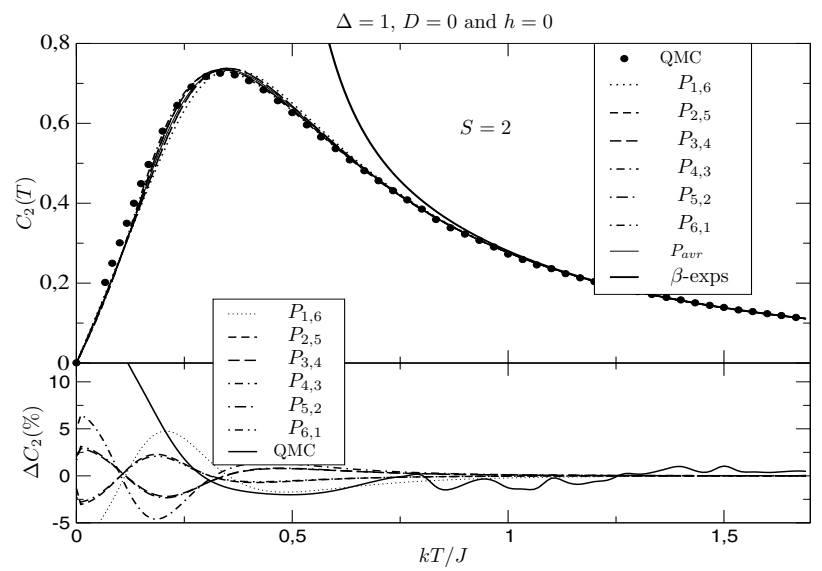

Fig. 6. (Top) The Padé approximant of the specific heat as a function of $k T / J$, for $S=2$ with $\Delta=1, D=0$ and $h=$ 0 . The thicker continuous line stands for the $\beta$-expansion of $C_{2}(T)$. (Bottom) The $\Delta C_{2}(T) 100 \%$ represents the percental deviation of all acceptable (non-singular) Padé approximants and the QMC results [21] with respect to the average of Padé approximants.

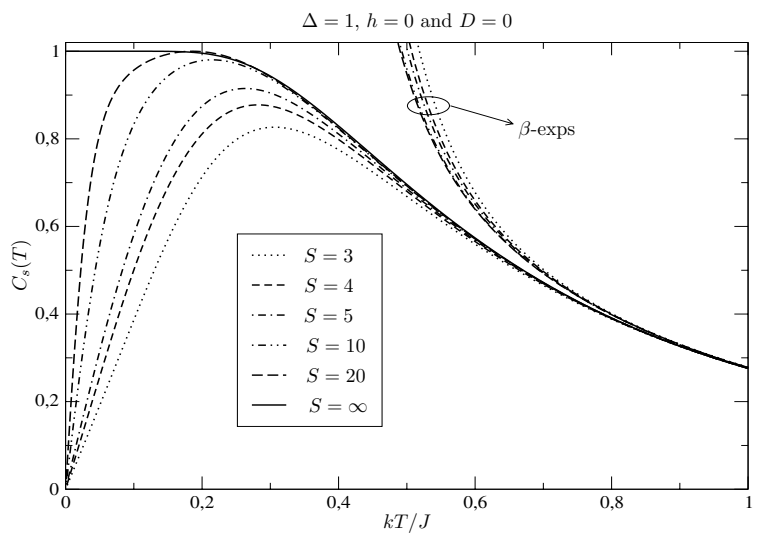

Fig. 7. The specific heat versus temperature for several values of spin at the isotropic point $\Delta=1$ with $h=0$ and $D=0$. For each value of spin, the curve is obtained by taking the average of all "acceptable" Padé approximants (i.e., with no spurious singularities), whereas in the classical limit corresponds to Fisher's solution.

approximant $P_{m, n}$ with respect to $P_{a v r}$. We define $\Delta C_{2}(\%)=\left(P_{m, n}-P_{a v r}\right) / P_{a v r} \times 100 \%$. The high temperature expansion satisfactorily describes $C_{2}(T)$ up to $k T / J \sim 0.9$. The solid line corresponds to the percental deviation of $P_{\text {avr }}$ with respect to the QMC prediction. Although we have only seven terms in expansion (B.3) of the Helmholtz free energy, the percental relative difference of the Padé approximants is smaller than 2,5\% for $k T / J \approx 0.25$, allowing good precision in calculating the temperature where $C_{2}(T)$ is maximum.

Figures 7 and 9 show the specific heat for several values of spin for the antiferromagnetic $(\Delta=1)$ and ferromagnetic $(\Delta=-1)$ cases, respectively, allowing us to observe how the specific heat per site approaches its classical limit 

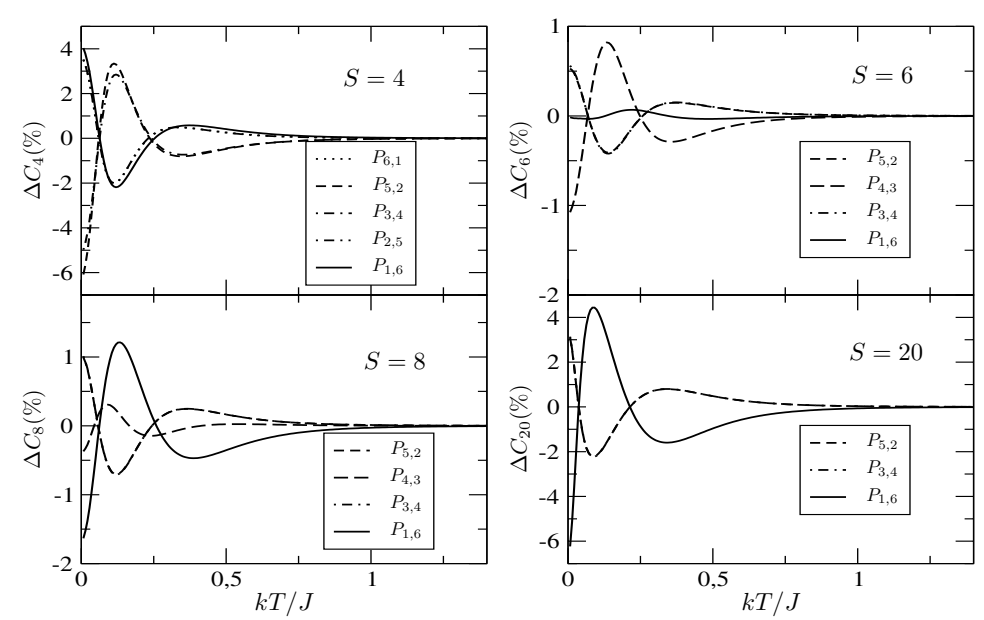

Fig. 8. The relative percental difference of all "acceptable" Padé's approximants to the average Padé $P_{a v r}$, for the specific heat per site for the antiferromagnetic case $(\Delta=1$ with $h=0$ and $D=0$ ), with $S=4,6,8$ and 20. Approximants with spurious singularities have been excluded.

as $S$ varies. In order to show how the Padé approximants enhance the high temperature solution of the specific heat per site, Figure 7 also displays the $\beta$-expansion of each $C_{s}(T)$. Following the spin wave theory, we assume that the specific heat behaves as $C_{s} \sim T$ for the antiferromagnetic [23] case, and as $C_{s} \sim T^{1 / 2}$ in the ferromagnetic [24] case. We fit the numerical values of the ground state energy per site calculated in reference [22], for the antiferromagnetic case with $S=1,2$ and 3 , and extrapolate them to the integer antiferromagnetic spin $S$ chain. Our approximate result for this ground state energy per site is $e_{0} / J \approx-\left(1+0.3641 / S+0.029 / S^{2}+0.0086 / S^{3}\right) S /(S+1)$. For the ferromagnetic case at the Heisenberg point $(\Delta \pm 1)$ with $h=0$ and $D=0$, the ground state energy simply becomes $e_{0} / J=-S /(S+1)$.

Figure 7 shows the specific heat as a function of temperature for $\Delta=1$, with $D=0$ and $h=0$. The curves correspond to the $P_{\text {avr }}$ of this thermodynamic function for the following set of spin values, as well as their respective $\beta$-expansions: $S=\{3,4,5,10,20\}$. The exact classical curve corresponds to Fisher's solution [10]. From our numerical results, we verify that even the Padé approximants for $S=3$ deviates less than $2 \%$ from the classical result $(S \rightarrow \infty)$ up to $k T / J \sim 0.64$, while for $S=5$ this deviation is about $2 \%$ only at $k T / J \sim 0.42$.

Except for the classical limit $(S \rightarrow \infty)$, to the best of our knowledge there are no known results of the specific heat per site for $S \geq 3$ for the whole range of temperature. In order to assure the validity of our results for the specific heat at lower temperatures, for distinct $S$ as presented in Figure 7, we plotted in Figure 8 the relative percental difference between the Padé approximants without spurious poles and the average Padé for several values of $\operatorname{spin}(S=4,6,8$ and 20$)$. For all those values of spin, it can be seen that deviations get larger than $2 \%$

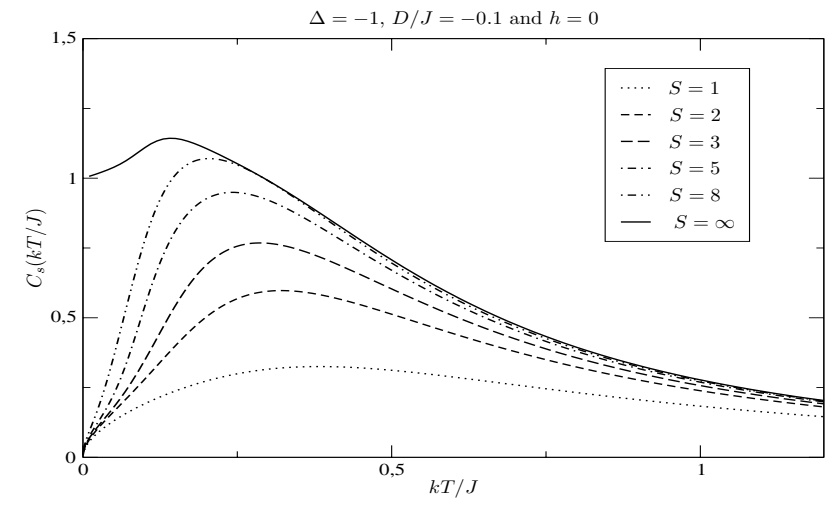

Fig. 9. The specific heat per site versus temperature $(k T / J)$ for several values of spin in the ferromagnetic case. We take: $\Delta=-1$ with $h=0$ and $D=-0.1$. Each curve is obtained by plotting its $P_{a v r}$, while the classical limit is obtained numerically.

for $k T / J \lesssim 0.25$, whereas their respective $\beta$-expansion are good approximations only up to $k T / J \sim 0.8$.

In Figure 7 we verify that the curves of the specific heat for the antiferromagnetic case are close to the classical curve up to low temperatures, even for $S=3$. Comparing its behavior to that of the ferromagnetic case, we plot in Figure 9 the specific heat for several values of spin, taking $\Delta=-1, h=0$ and $D=-0.1$. We assume that the specific heat for the ferromagnetic case and small values of $D$ still behaves as $C_{L} \sim T^{1 / 2}$ and the ground state energy per site is $e_{0} / J=-S /(S+1)-D S /(S+1)-D / 3$. The first term in the ground state energy corresponds to the case $D=0$; the second one is the contribution of the single-ion anisotropy to the ground state energy; and the last term corresponds to its global shift. In order to simplify our 

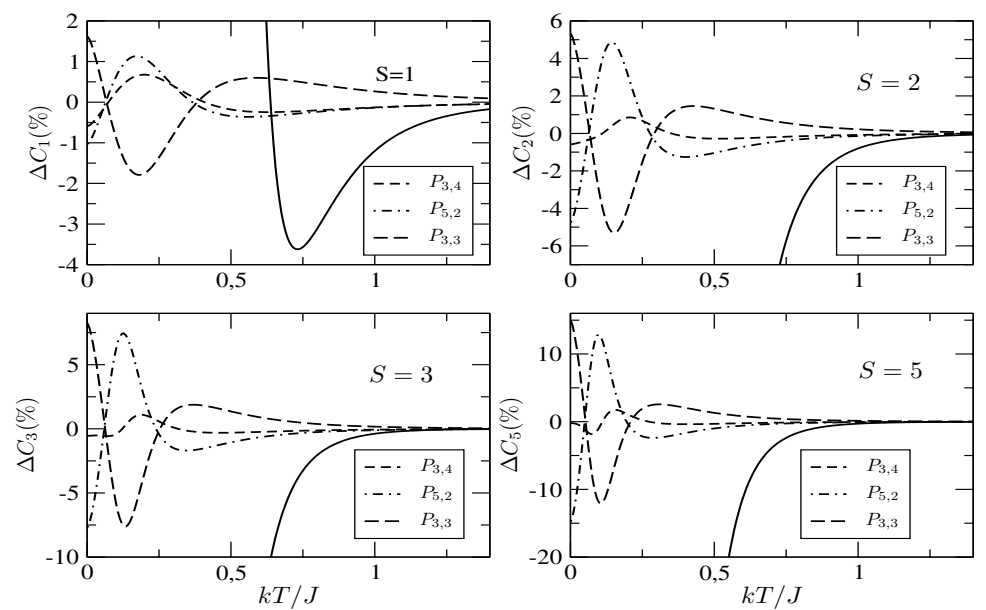

Fig. 10. The relative percental difference of the Padé's approximants to the average Padé $P_{a v r}$ of the specific heat per site for the antiferromagnetic case $(\Delta=1$ with $h=0$ and $D=0)$, for $S=4,6,8$ and 20. Approximants with spurious singularities have been excluded. The continuous line in each picture represents the percental difference between the $\beta$-expansion of $C_{s}(T)$ and $P_{a v r}$.

calculations, we impose that the average energy vanishes at $\beta=0$ (otherwise it would be equal to $D / 3$, that is, the value obtained from our high temperature expansion).

As in the antiferromagnetic case, we do not know of results that would allow comparison regarding the dependence of the specific heat on the temperature in the interval $[0, \infty]$. In order to carry out a first check of the curves in Figure 9, we proceed in a similar way as before when testing the antiferromagnetic curves. Figure 10 shows the relative percental differences of the Padé's approximants and the respective $\beta$-expansion of $C_{s}(T)$ to the average Padé for $S=1,2,3$ and 5 . We see that the high temperature series describe well the specific heat of the ferromagnetic chains for $k T / J \gtrsim 1$. From theses plots we verify that the extension of the high-temperature results to the region of lower temperatures for the ferromagnetic case is worse than that of the antiferromagnetic case, even though integer-spin ferromagnetic materials are gapless and the behavior of their specific heat about $T=0$ is well described by the spin-wave approach.

It is also possible to write the magnetic susceptibility per site as a function of the ground state energy per site $e$. Its low temperature behavior can be inferred from the extrapolation in a similar fashion as that of the specific heat per site. Unfortunately, convergence is not as much as satisfactory. By applying the method of reference [14] to the magnetic susceptibility, a large number of singularities are found in the Padé approximants to this thermodynamic function. For this reason we use the Dlog-Padé approximant [15] to extend the high temperature expansion of the antiferromagnetic susceptibility, incorporating the low-temperature information in a simple way. We follow reference [15] to calculate the Dlog-Padé approximant of the magnetic susceptibility per site $\chi(\beta)$; we refer the reader to this article for further details on the method.

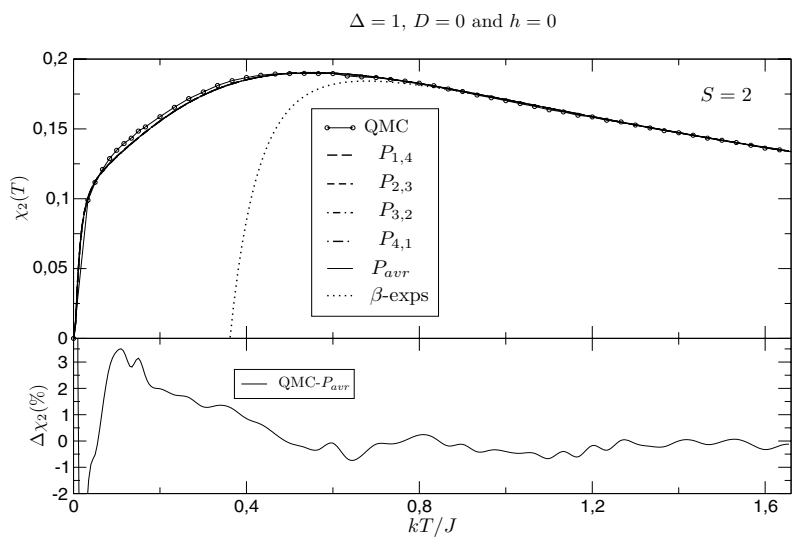

Fig. 11. The magnetic susceptibility for the antiferromagnetic case $\Delta=1, D=0$ and $h=0$. a) Dlog-Padé approximants of $\chi(T)$ versus $k T / J$ are compared to QMC results [21]. b) the average Dlog-Padé approximant of $\chi(T)$ as a function of $k T / J$ for various spin values.

From reference [15], we verify that the Dlog-Padé approximants are independent of the leading term coefficient of the magnetic susceptibility at low temperatures, if it vanishes at $T \rightarrow 0$. On the other hand, if it is non-null at $T=0$, we need to know the exact coefficient of the leading term of the magnetic susceptibility for $T \sim 0$ in order to calculate the Padé approximants at low temperatures. This also occurs [25] for $S=1 / 2$.

Using the QMC method, Yamamoto [21] obtained the magnetic susceptibility of the antiferromagnetic $S=2$ $X X Z$ model $(\Delta=1)$ in the whole interval of temperature of a periodic chain with 96 sites. In Figure 11 we compare the $\beta$-expansion of $\chi_{2}(T)$ and its Dlog-Padé approximants 

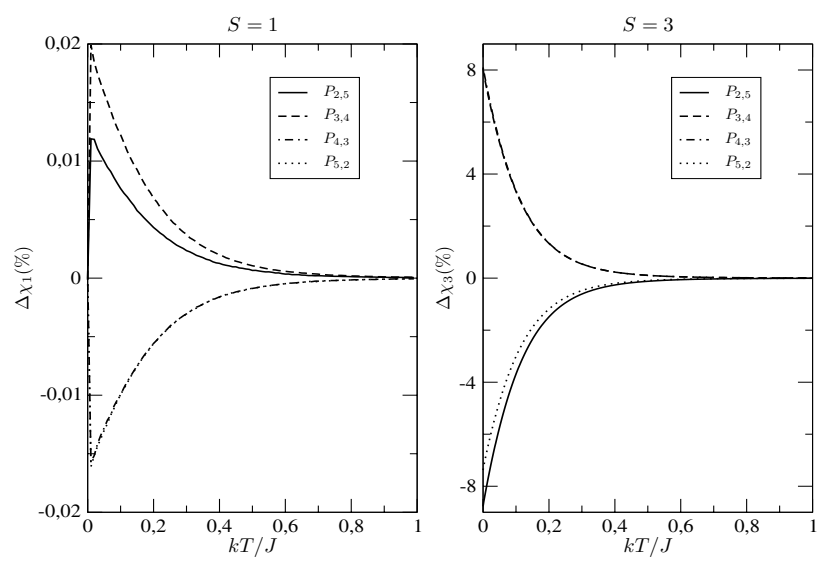

Fig. 12. The percental difference between the magnetic susceptibility of the D-log Padé approximants and $P_{\text {avr }}$ for the antiferromagnetic model $(D=0)$, with $S=1$ and $S=3$, and no external magnetic fields.

for $S=2$, with $\Delta=1, D=0$ and $h=0$ with Yamamoto's numerical results. Our extension to lower temperatures assumes that the behavior of the magnetic susceptibility around $T=0$ be as $T^{\alpha} \exp \left(-E_{g a p} / k T\right)$ with $\alpha \approx-1 / 4$; the latter value yields the best fit to the QMC results [21]. The bottom of Figure 11 shows the percental difference between the QMC and the average Dlog-Padé approximant to the magnetic susceptibility for $S=2$; it can be seen that $\Delta \chi_{2}(\%) \leq 2 \%$ up to $k T / J \approx 0.2$. From Figure 11 (top) we verify that our high-temperature expansion of $\chi_{2}(T)$ fits well the numerical result only up to $k T / J \sim 0.7$.

Figure 12 shows the deviation from the average $P_{a v r}$ of the Dlog-Padé approximants to the magnetic susceptibility, for the antiferromagnetic Heisenberg model $(\Delta=1$ and $D=0$ ) for $S=1$ and $S=3$, showing the enhanced extention of our high-temperature results to lower temperatures. From reference [20] we obtain the behavior of $\chi$ around $T=0(\alpha=0.5)$; for $S=3$ we assume $\alpha \approx 0$.

The behavior of the magnetic susceptibility at low temperature for the ferromagnetic Heisenberg model $(\Delta=-1$ and $D=0$ ) was obtained by Takahashi [24] using the modified spin wave theory for arbitrary spin-S. The magnetic susceptibility for the unitary spin chain for low temperatures, in units of $k$, is

$$
\chi_{s}(T)=2\left(\frac{S}{S+1}\right)^{2} T^{-2}\left(\frac{1}{3}-a T^{1 / 2}+a^{2} T+\mathcal{O}\left(T^{3 / 2}\right)\right),
$$

where $a=\frac{\zeta\left(\frac{1}{2}\right)}{2 S} \sqrt{\frac{S+1}{\pi}}$ and $\zeta(x)$ is the Riemann zeta function. When the Dlog-Padé approximant method [15] is applied, not even the coefficient of the leading term of equation (9) is used, but only its power. Therefore, a better approximation will be achieved if we take into account all the three terms in (9); we do so by applying the twopoint Padé approximant [16] method.

We verify from expansion (9) that $\chi_{s}(T)$ is singular at $T=0$. Expansion (B.3) gives the $\beta$-expansion of $\chi_{s}(\beta)$

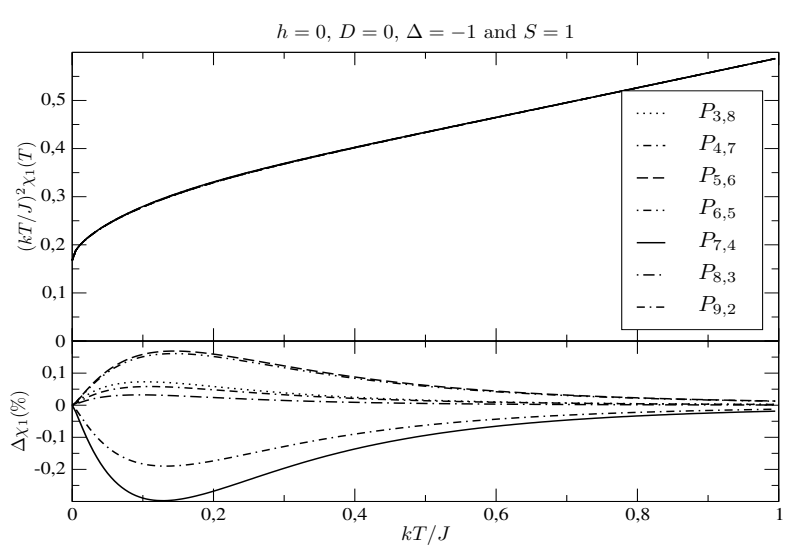

Fig. 13. (Top) The function $(k T / J)^{2} \chi_{1}(T)$ versus $k T / J$ of the isotropic ferromagnetic $S=1$ model $(\Delta=-1$ and $D=0)$ in the absence of external magnetic fields, for $P_{a v r}$. (Bottom) The relative percental difference of non-singular Padé approximants to $P_{\text {avr }}$.

up to order $\beta^{6}$. The first terms of $\chi_{s}(\beta)$ for $\Delta=-1$, $D=0$ and $h=0$ are $\chi_{s}(\beta) \approx \beta / 3+2 \beta^{2} / 9$. We define the auxiliary function $\mathcal{P}_{s}(\beta) \equiv \frac{\chi_{s}(\beta)}{\beta^{2}}-\frac{1}{3 \beta}$. This auxiliary function is regular at $T=0$ and $T \rightarrow \infty(\beta=0)$; its only problems are that it has non-integer powers of $\beta$ and it does not have a Taylor expansion about $T=0$. In order to circumvent this drawback, we proceed as in reference [15] and apply the transformation

$$
u=\beta^{1 / 2} /\left(1+\beta^{1 / 2}\right) \Leftrightarrow \beta=u^{2} /(1-u)^{2},
$$

where the parameter $\beta \in[0, \infty\rangle$ is mapped onto $u \in[0,1]$. The expansions of $\mathcal{P}$, around $T=0(u=1)$ and $\beta=$ $0(u=0)$, are polynomials in $u$ and therefore we can write its two-point Padé's approximants, connecting the expansions of $\mathcal{P}_{s}$ at $u=1$ and $u=0$.

Figure 13 shows the average Padé of the magnetic susceptibility for the isotropic ferromagnetic $S=1$ model, in the absence of an external magnetic field, obtained from the two-point Padé method and using the transformation (10). For all Padé approximants, the relative difference to $P_{\text {avr }}$ is smaller than $0.3 \%$ in the whole interval of temperature.

Finally, Figure 14 shows the relative percental difference of various Padé approximants to the $P_{a v r}$, in the case of the ferromagnetic isotropic chain model (with $D=0$ ) and in the absence of an external magnetic field. The difference increases with $S$; however, for $S=5$ the relative difference is smaller than $2 \%$ up to $k T / J \approx 0.3$.

\section{Conclusions}

In this report we present the high temperature expansion of the Helmholtz free energy of the $X X Z$ chain, in the thermodynamic limit, for an arbitrary spin $S$ up to order $\beta^{6}$. This expansion is analytic in $S$ and in $\beta$ and each coefficient of $\beta^{n}(n=-1,0,1, \cdots 6)$ is exact. The model includes anisotropy in the $z$-direction, single-ion anisotropy 

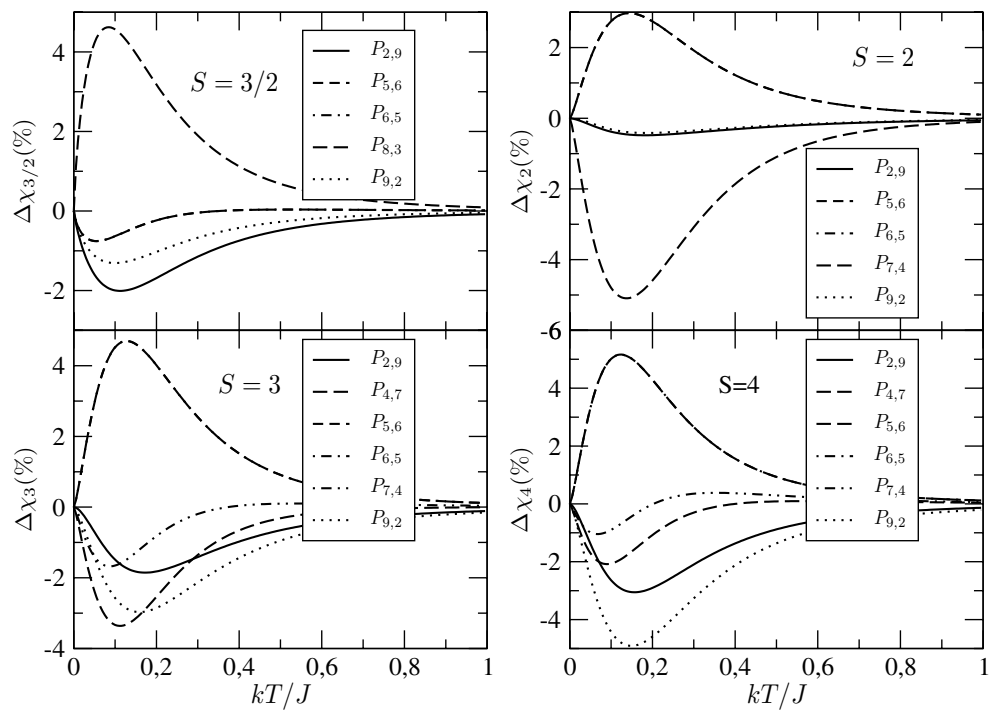

Fig. 14. The relative percental difference between the Padé approximants to the magnetic susceptibility (ferromagnetic case) and $P_{\text {avr }}$ for $S=3 / 2,2,3$ and 4 . Here, $\Delta=-1, D=0$ and $h=0$.

and an external magnetic field. In order to obtain the expansion of the Helmholtz free energy (B.3) we apply the method developed in reference [13]; from this expansion we are able to obtain the thermodynamic quantities of the model for arbitrary spin values. The expansion (B.3) recovers the $\beta$-expansion of the Helmholtz free energy presented in Reference [9] for several values of spin, and it is an extension to the results of Reference [12], where we include the anisotropy effects.

The series in $S$ allows us to obtain the classical limit of the $X X Z$ model, but taking into account the quantum nature of the spins. For the particular case $h=0$ and $D=0$ we recover Joyce's result for the classical limit of this model [11]. We explicitly show in expansion (4) that only the presence of an external magnetic field distinguishes the classical ferromagnetic and antiferromagnetic phases in the regime of high temperatures.

We interpolate the $\beta$-expansion with its low temperature series using the Padé approximant of (B.3) to extrapolate the properties of the specific heat to finite temperatures using the method developed in reference [14]. Only a few terms of the high temperature series are needed to yield good agreement: for low spin values the deviation of all non-singular Padé approximants respect to $P_{a v r}$ fluctuates around $5 \%$.

For the antiferromagnetic magnetic susceptibility we use the Dlog-Padé [15] approximants to investigate the finite temperature properties of the spin- $s$ quantum XXZ chain. In order to plot the magnetic susceptibility $\chi_{s}(T)$ as a function of temperature for different spin values we take the average of all non-singular Dlog-Padé approximants, since their deviation from the corresponding $P_{a v r}$ is less than $2 \%$. The ferromagnetic case is studied by applying two-point like Padé approximants, since we have the first three terms of the low temperature series expansion [24]. The magnetic susceptibility is plotted for different spins, in the same way as for the previous case.

Obviously, if we knew more low temperature information about any physical quantities, it could be also interpolated with our high temperature expansions, by adapting different approximants available in the literature. Our main limitation regarding the study of finite temperature behavior of other parameters of the Hamiltonian (2) is the lack of related low temperature information.

O.R. thanks FAPEMIG for financial support. The authors are in debt to CNPq for partial financial support. S.M. de S. thanks to FAPEMIG and M.T.T thanks FAPERJ for partial financial support.

\section{Appendix A: Normalized traces of $\left(\mathrm{S}^{z}\right)^{l}$}

We have that $\operatorname{tr}\left(\left(S^{z}\right)^{n}\right)=0$ if $n$ is odd. For the purposes of this appendix, $n$ is taken as an even integer. In order to calculate $\operatorname{tr}\left(\left(S^{z}\right)^{n}\right)$ we use the result of reference [17] that permits us to write this trace as a function of the spin $S$,

$$
\operatorname{tr}\left(\left(S^{z}\right)^{n}\right)=\sum_{j=-S}^{S} j^{n}=\sum_{r=0}^{n+1} C_{r, n} S^{r},
$$

where $S=1 / 2,1,3 / 2, \cdots, \quad C_{r, n}=$ $\frac{\left(1+(-1)^{n}\right)}{n+1} \sum_{k=0}^{n+1}\left(\begin{array}{l}k \\ r\end{array}\right) b_{k, n+1}$, so that $b_{k, n}$ 's are the coefficients of Bernoulli's polynomial $B_{n}(x)$. They are defined by $[17]$

$$
B_{n}(x)=\sum_{k=0}^{n} b_{k, n} x^{k}
$$


On the other hand, we prefer to rewrite equation (A.1) as a function of the square of the norm of the spin $\mathbf{S}$ (that is, $S(S+1)$ ), which is a constant of motion,

$$
\sum_{j=-S}^{S} j^{n}=(2 S+1) \sum_{r=0}^{n / 2} A_{r, n}(S(S+1))^{r}
$$

We remind that $2 S+1$ is equal to the dimension of the Hilbert space at each site, that is, $\operatorname{tr}_{i}(\mathbf{1})=2 S+1$, $i=1,2, \cdots, N$. Comparing equations (A.1) and (A.3), and after some algebraic manipulation, we obtain each coefficient $A_{r, n}$ of equation (A.3) as a combination of the Bernoulli numbers [17] $B_{n+1-j}$,

$A_{k, n}=\frac{\left(1+(-1)^{n}\right)(-1)^{k+1}}{n+1} \sum_{j=0}^{k}\left(\begin{array}{c}n+1 \\ j\end{array}\right)\left(\begin{array}{c}2 k-j \\ k\end{array}\right) B_{n+1-j}$.

In equation (2) we rewrite Hamiltonian (1) in terms of the rescaled spin operator $\mathbf{s}$. To obtain the results (B.4-B.10) we need to calculate the powers of the operator $s^{z}$. From the results (A.1-A.4) we are able to write the normalized traces of $s^{z}$, that is $\left\langle\left(s^{z}\right)^{2 n}\right\rangle$ just as a polynomial of $y \equiv \frac{1}{S(S+1)}$ of degree $n / 2-1$, which reads

$$
\left\langle\left(s^{z}\right)^{n}\right\rangle=\sum_{r=0}^{n / 2} A_{n / 2-r, n} y^{r}
$$

In terms of the normalized matrix representation, the commutation relation becomes

$$
\left[s^{x}, s^{y}\right]=\frac{i s^{z}}{\sqrt{S(S+1)}}=i \sqrt{y} s^{z},
$$

which is useful to evaluate the normalized traces.

\section{Appendix B: The $\beta$-expansion of the free energy for the spin-s $X X Z$ chain}

We call $\mathcal{W}_{s}(\beta)$ the Helmholtz free energy of the Hamiltonian (2), that drives the dynamics of the quantum spin chain with unitary norm. Let $\mathcal{W}_{S}(\beta)$ be the Helmholtz free energy of the Hamiltonian

$$
\mathbf{H}_{S}=\sum_{i=1}^{N} J\left(\mathbf{S}_{i}, \mathbf{S}_{i+1}\right)_{\Delta}-h S_{i}^{z}+D\left(S_{i}^{z}\right)^{2}
$$

where the norm of the spin-S is $\sqrt{S(S+1)}$ and $S=$ $1 / 2,1,3 / 2, \cdots$. In reference [9] we calculated the high temperature expansion of the Helmholtz free energy of the Hamiltonian (B.1) up to $S=4$. The relation between the free energies derived from Hamiltonians (2) and (B.1) is

$$
\begin{aligned}
\mathcal{W}_{s}(J, \Delta, h, D ; \beta)=\frac{1}{S(S+1)} \\
\quad \times \mathcal{W}_{S}\left(J, \Delta, \sqrt{S(S+1)} h, D ; \frac{\beta}{S(S+1)}\right) \quad(\mathrm{B} .2 \mathrm{a}) \\
=\mathcal{W}_{S}\left(\frac{J}{S(S+1)}, \Delta, \frac{h}{\sqrt{S(S+1)}}, \frac{D}{S(S+1)} ; \beta\right) .
\end{aligned}
$$

In equations (B.2), the parameters of the respective Hamiltonians are shown explicitly. Equating equations (B.2a) and (B.2b), we verify that $\mathcal{W}_{S}$ is an homogeneous function of degree 1 .

In this appendix we present the high temperature expansion of the Helmholtz free energy $\mathcal{W}_{s}(\beta)$, up to order $\beta^{6}(n=7)$, for arbitrary value of $s(s=S)$. This expansion can be written in terms of $y \equiv \frac{1}{S(S+1)}$, that is,

$$
\mathcal{W}_{s}(\beta)=-\frac{\ln (2 S+1)}{\beta}+\sum_{r=0}^{6} w_{r}(y) \beta^{r}+\mathcal{O}\left(\beta^{7}\right)
$$

The coefficients $w_{r}(y)$, for $r=0,1, \cdots, 6$, are shown below. For the sake of simplicity, we define $x \equiv(3 y-4)$.

$$
\begin{gathered}
w_{0}(y)=\frac{D}{3} \\
w_{1}(y)=\frac{D^{2} x}{90}-\frac{h^{2}}{6}-\frac{J^{2}}{9}-\frac{J^{2} \Delta^{2}}{18}
\end{gathered}
$$

$$
\begin{aligned}
w_{2}(y) & =\left(\frac{J^{2} D}{135}+\frac{D^{3}(15 y-4)}{5670}-\frac{J^{2} \Delta^{2} D}{135}-\frac{h^{2} D}{90}\right) x \\
& -\frac{J^{3} \Delta y}{36}+\frac{J \Delta h^{2}}{9}
\end{aligned}
$$

$$
\begin{aligned}
w_{3}(y) & =\left(\frac{2 J \Delta h^{2} D}{135}-\frac{h^{2} D^{2}(15 y-4)}{3780}-\frac{J^{2} D^{2}(27 y+4)}{18900}\right. \\
& \left.+\frac{D^{4}\left(105 y^{2}-54 y-8\right)}{113400}-\frac{8 J^{2} \Delta^{2} D^{2}(2 y-1)}{4725}\right) x \\
& +\frac{h^{4}(y+2)}{360}-\frac{J^{4}\left(-128 y+12+33 y^{2}\right)}{16200}+\frac{J^{2} \Delta^{2} h^{2}(3 y-14)}{270} \\
& -\frac{J^{4} \Delta^{4}\left(-16 y+14+y^{2}\right)}{5400} \\
& +\frac{J^{2} h^{2}(9 y+8)}{540}-\frac{J^{4} \Delta^{2}\left(-32 y-72+27 y^{2}\right)}{8100}
\end{aligned}
$$

See equation (B.8) next page

See equation (B.9) next page

See equation (B.10) next page

The coefficients $J, D$ and $h$ in (B.4)-(B.10) are the constants in Hamiltonian (2).

Writing the expansion (B.3) in terms of the variable $y$ greatly simplifies the calculation of its classical limit $(y \rightarrow 0)$. 


$$
\begin{aligned}
w_{4}(y)= & \left(\frac{J^{2} \Delta^{2} h^{2} D(16 y-43)}{4725}+\frac{16 J \Delta h^{2} D^{2}(2 y-1)}{4725}-\frac{J^{4} \Delta^{4} D\left(y^{2}-12 y+4\right)}{11340}-\frac{h^{2} D^{3}\left(105 y^{2}-54 y-8\right)}{56700}\right. \\
& -\frac{J^{3} \Delta \mathrm{D}^{2} y(9 y-2)}{8100}+\frac{D^{5}(15 y-4)\left(105 y^{2}-48 y-16\right)}{3742200}-\frac{J^{4} \Delta^{2} D(3 y+4)(y-4)}{22680}+\frac{J^{2} h^{2} D(27 y+4)}{18900} \\
& \left.+\frac{J^{4} D\left(5 y^{2}-32 y-8\right)}{22680}+\frac{J^{2} D^{3}\left(3 y^{2}-60 y-32\right)}{170100}+\frac{h^{4} D(5 y+8)}{7560}-\frac{J^{2} \Delta^{2} D^{3}\left(75 y^{2}-63 y+4\right)}{42525}\right) x \\
& -\frac{J \Delta h^{4}(y+2)}{135}+\frac{J^{3} \Delta h^{2}(9 y+8)(y-3)}{1350}+\frac{J^{3} \Delta^{3} h^{2}\left(-48 y+92+3 y^{2}\right)}{4050}-\frac{J^{5} \Delta^{3} y\left(-16 y-4+3 y^{2}\right)}{6480} \\
& -\frac{J^{5} \Delta y\left(-16 y-4+3 y^{2}\right)}{3240}
\end{aligned}
$$

$$
\begin{aligned}
w_{5}(y)= & -\frac{J^{2} D^{4}\left(65223 y^{3}+34020 y^{2}-5904 y-16064\right)}{785862000}+\frac{J^{3} \Delta^{3} h^{2} D\left(3 y^{2}-36 y+40\right)}{8505}+\frac{J^{5} \Delta D y^{2}(4 y-27)}{40500} \\
& -\frac{J^{4} D^{2}\left(4287 y^{3}-14910 y^{2}+2404 y+3184\right)}{23814000}+\frac{J^{3} \Delta h^{2} D\left(39 y^{2}-62 y-40\right)}{28350}-\frac{2 J \Delta h^{4} D(16 y+27)}{14175} \\
& +\frac{2 J \Delta h^{2} D^{3}\left(75 y^{2}-63 y+4\right)}{42525}-\frac{J^{3} \Delta D^{3} y\left(213 y^{2}-129 y+20\right)}{425250}+\frac{J^{2} h^{2} D^{2}\left(123 y^{2}+32 y+32\right)}{113400} \\
& +\frac{D^{6}\left(7182945 y^{4}-6404130 y^{3}+569016 y^{2}+443424 y+2944\right)}{30648618000}-\frac{h^{2} D^{4}(15 y-4)\left(105 y^{2}-48 y-16\right)}{1496880} \\
& -\frac{J^{4} \Delta^{4} D^{2}\left(111 y^{3}-1155 y^{2}+888 y-16\right)}{1190700}+\frac{h^{4} D^{2}\left(105 y^{2}+74 y-72\right)}{226800}+\frac{J^{2} \Delta^{2} h^{2} D^{2}\left(15 y^{2}-36 y+16\right)}{5670} \\
& -\frac{J^{4} \Delta^{2} D^{2}\left(369 y^{3}-546 y^{2}-751 y-116\right)}{1488375}-\frac{J^{2} \Delta^{2} D^{4}\left(204885 y^{3}-224028 y^{2}+39888 y+8768\right)}{196465500} \\
& \left.-\frac{J^{5} \Delta^{3} D y^{2}(4 y-27)}{40500}\right) x+\frac{J^{4} \Delta^{4} h^{2}\left(-768 y^{2}-2872+2748 y+45 y^{3}\right)}{340200}-\frac{h^{6}\left(12 y+16+3 y^{2}\right)}{45360} \\
& -\frac{J^{2} h^{4}\left(632 y+432+333 y^{2}\right)}{226800}-\frac{J^{6} \Delta^{2}\left(101120+203200 y+83481 y^{2}-289584 y^{3}+42174 y^{4}\right)}{142884000} \\
& -\frac{J^{6}\left(-33760-1920 y+239418 y^{2}-127872 y^{3}+14067 y^{4}\right)}{214326000}-\frac{J^{2} \Delta^{2} h^{4}\left(-424 y-1074+69 y^{2}\right)}{56700} \\
& -\frac{J^{6} \Delta^{4}\left(-16960+50720 y+30846 y^{2}-33624 y^{3}+4239 y^{4}\right)}{71442000}+\frac{J^{4} h^{2}\left(-108 y^{2}-32-160 y+27 y^{3}\right)}{45360} \\
& -\frac{J^{6} \Delta^{6}\left(1712-15408 y+14688 y^{2}-2700 y^{3}+135 y^{4}\right)}{42865200}+\frac{J^{4} \Delta^{2} h^{2}\left(-813 y^{2}+848+383 y+135 y^{3}\right)}{85050}
\end{aligned}
$$

$$
\begin{aligned}
& w_{6}(y)=-\frac{2 J \Delta h^{4} D^{2} x^{2}}{4725}+\left(-\frac{J^{2} \Delta^{2} D^{5}\left(1780695 y^{4}-2280645 y^{3}+677964 y^{2}+89296 y-25024\right)}{2554051500}\right. \\
& +\frac{J^{2} h^{2} D^{3}\left(262035 y^{3}-85176 y^{2}+12576 y-16064\right)}{392931000}-\frac{J^{6} \Delta^{6} D\left(45 y^{4}-720 y^{3}+2988 y^{2}-1608 y-208\right)}{15309000} \\
& -\frac{J^{2} h^{4} D\left(31 y^{2}+40 y+16\right)}{75600}-\frac{J^{4} \Delta^{2} D^{3}\left(25002 y^{4}-47646 y^{3}-23907 y^{2}+15308 y+10464\right)}{147349125} \\
& -\frac{J^{6} \Delta^{4} D\left(729 y^{4}-5328 y^{3}+4488 y^{2}+7784 y+1248\right)}{30618000}+\frac{J^{4} h^{2} D\left(1935 y^{3}-1974 y^{2}+2404 y+3184\right)}{23814000} \\
& +\frac{J^{6} \Delta^{2} D\left(108 y^{4}-684 y^{3}+201 y^{2}+524 y+624\right)}{15309000}+\frac{J^{2} \Delta^{2} h^{2} D^{3}\left(40977 y^{3}-91791 y^{2}+58890 y-7240\right)}{19646550} \\
& +\frac{J^{3} \Delta h^{2} D^{2}\left(7425 y^{3}-11907 y^{2}-2928 y-928\right)}{5953500}+\frac{J^{4} \Delta^{2} h^{2} D\left(972 y^{3}-4851 y^{2}+1567 y+2084\right)}{1786050} \\
& -\frac{J^{2} \Delta^{2} h^{4} D\left(35 y^{2}-141 y-332\right)}{56700}+\frac{J \Delta h^{2} D^{4}\left(204885 y^{3}-224028 y^{2}+39888 y+8768\right)}{98232750} \\
& -\frac{J^{5} \Delta^{3} D^{2} y\left(1422 y^{3}-8253 y^{2}+3697 y-208\right)}{11907000}-\frac{J^{5} \Delta D^{2} y\left(1455 y^{3}-5418 y^{2}-428 y+376\right)}{11907000} \\
& +\frac{J^{4} \Delta^{4} h^{2} D\left(1665 y^{3}-22113 y^{2}+53808 y-35212\right)}{17860500}+\frac{h^{4} D^{3}\left(2625 y^{3}+300 y^{2}-1928 y+224\right)}{7484400} \\
& +\frac{J^{3} \Delta^{3} h^{2} D^{2}\left(333 y^{3}-3465 y^{2}+4596 y-1504\right)}{893025}+\frac{J^{6} D\left(603 y^{4}-5400 y^{3}+10062 y^{2}+3520 y-416\right)}{30618000} \\
& -\frac{J^{4} \Delta^{4} D^{3}(2 y-1)\left(8925 y^{3}-79200 y^{2}+49076 y+8912\right)}{196465500}-\frac{J^{3} \Delta D^{4} y\left(4413 y^{3}-4214 y^{2}+724 y+48\right)}{11907000} \\
& -\frac{J^{2} D^{5}\left(150639 y^{4}+58088 y^{3}-28064 y^{2}-67200 y-17664\right)}{3405402000}-\frac{h^{6} D\left(7 y^{2}+22 y+24\right)}{226800} \\
& -\frac{J^{4} D^{3}\left(6201 y^{4}+346320 y^{3}-363672 y^{2}-223664 y-35136\right)}{2357586000}-\frac{J \Delta h^{4} D^{2} y^{2}}{567} \\
& +\frac{D^{7}(15 y-4)\left(315315 y^{4}-238140 y^{3}+1896 y^{2}+17664 y+2944\right)}{30648618000} \\
& \left.-\frac{h^{2} D^{5}\left(7182945 y^{4}-6404130 y^{3}+569016 y^{2}+443424 y+2944\right)}{10216206000}\right) x+\frac{J \Delta h^{6}\left(44 y+54+11 y^{2}\right)}{28350} \\
& -\frac{J^{7} \Delta y\left(1600+6432 y+51631 y^{2}-24768 y^{3}+2556 y^{4}\right)}{40824000}-\frac{J^{3} \Delta h^{4},\left(-284 y^{2}-640-834 y+99 y^{3}\right)}{113400} \\
& -\frac{J^{7} \Delta^{5} y\left(-400-3344 y+9228 y^{2}-2964 y^{3}+243 y^{4}\right)}{20412000}-\frac{J^{3} \Delta^{3} h^{4}\left(-228 y^{2}+1488+328 y+15 y^{3}\right)}{85050} \\
& +\frac{J^{5} \Delta^{3} h^{2}\left(-33440+1080 y+51411 y^{2}-25974 y^{3}+2754 y^{4}\right)}{7144200}+\frac{J^{5} \Delta h^{2}\left(10112+30512 y+4629 y^{2}-20844 y^{3}+3159 y^{4}\right)}{7144200} \\
& +\frac{J^{5} \Delta^{5} h^{2}\left(20528-31872 y+16452 y^{2}-2700 y^{3}+135 y^{4}\right)}{7144200}-\frac{J^{7} \Delta^{3} y\left(3200+19808 y+14719 y^{2}-12912 y^{3}+1584 y^{4}\right)}{20412000}
\end{aligned}
$$




\section{References}

1. A. Klümper, Z. Phys. B 91, 507 (1993); A. Klümper, Eur. Phys. J. B 5, 687 (1998)

2. A.P. Tonel, A. Foerster, J. Links, A.L. Malvezzi, Phys. Rev. B 64, 054420 (2001), and references therein

3. H. Kadowaki, K. Hirakawa, K. Ubukoshi, J. Phys. Soc. Jpn 52, 1799 (1983)

4. S. Itoh, Y. Endoh, K. Kakurai, H. Tanaka, Phys. Rev. Lett. 74, 2375 (1995)

5. S. Itoh, Y. Enodh, K. Kakurai, S.M. Bennington, T.G. Perring, K. Ohoyama, M.J. Harris, K. Nakajima, C.D. Frost, Phys. Rev B 59, 14406 (1999)

6. G.E. Granroth, M.W. Meisel, M. Chaparala, Th. Jolicour, B.H. Ward, D.R. Taham, Phys. Rev. Lett. 77, 1616 (1996)

7. R.J. Birgeneau, R. Dingle, M.T. Hutchings, G. Shirane, S.L. Holt, Phys. Rev. Lett. 26, 718 (1971)

8. M.T. Hutchings, G. Shirane, R.J. Birgeneau, S.L. Holt, Phys. Rev. B 5, 1999 (1972)

9. O. Rojas, S.M. de Souza, E.V. Corrêa Silva, M.T. Thomaz, J. Phys.: Condens. Matt. 15, 8527 (2003)

10. M.E. Fisher, Am. J. Phys. 32, 343 (1964); T. Delica, H. Leschke, Phys. A 168, 768 (1990)

11. G.S. Joyce, Phys. Rev. Lett. 19, 581 (1967)
12. N. Fukushima, A. Honecker, W. Brening, Phys. Rev. B 69, 174430 (2004)

13. O. Rojas, S.M. de Souza, M.T. Thomaz, J. Math. Phys. 43, 1390 (2002)

14. B. Bernu, G. Misguich, Phys. Rev. B 63, 134409 (2001)

15. A. Bühler, U. Löw, G.S. Uhrig, Phys. Rev. B 64, 024428 (2001)

16. G.A. Baker Jr., G.S. Rushbrooke, H.E. Gilbert, Phys. Rev. 135, A1272 (1964)

17. M. Abramowitz, I.A. Segun, Handbook of Mathematical Functions (Dover Publ., N.Y. 1964)

18. S.J. Collocott, R. Driver, L. Dale, S.X. Dou, Phys. Rev. B 37, 7917 (1988)

19. F.D.M. Haldane, Phys. Lett. A 93, 464 (1983); F.D.M. Haldane, Phys. Rev. Lett. 50, 1153 (1983)

20. T. Jolicoeur, O. Golinelli, Phys. Rev. B50, 9265 (1994)

21. S. Yamamoto, Phys. Rev. B 53, 3364 (1996); S. Yamamoto, Phys. Lett. A 213, 102 (1996)

22. S. Todo, K. Kato, Phys. Rev. Lett. 87, 047203 (2001)

23. R. Kubo, Phys. Rev. 87, 568 (1952)

24. M. Takahashi, Phys. Rev. Lett. 58, 168 (1987)

25. S. Eggert, I. Affleck, M. Takahashi, Phys. Rev. Lett. 73, 332 (1994) 